7. - Converse theorems of summability for Dirichlet's series, Trans. Amer. Math. Soc. vol. 39 (1936) pp. 117-130. 1946.

8. D. V. Widder, The Laplace transform, Princeton Mathematical Series, no. 6 ,

Madras Christian College

\title{
TYPICALLY-REAL FUNCTIONS WITH ASSIGNED ZEROS
}

\section{A. W. GOODMAN}

1. Introduction. A function $f(z)$

$$
f(z)=\sum_{n=0}^{\infty} b_{n} z^{n}
$$

is said to be typically-real of order $p$, if in (1.1) the coefficients $b_{n}$ are all real and if either (I) $f(z)$ is regular in $|z| \leqq 1$ and $\Im f\left(e^{i \theta}\right)$ changes sign $2 p$ times as $z=e^{i \theta}$ traverses the boundary of the unit circle, or (II) $f(z)$ is regular in $|z|<1$ and if there is a $\rho<1$ such that for each $r$ in $\rho<r<1, \Im f\left(r e^{i \theta}\right)$ changes sign $2 p$ times as $z=r e^{i \theta}$ traverses the circle $|z|=r$. This set of functions is denoted by $T(p)$.

The name typically-real was first suggested by Rogosinski [6] ${ }^{1}$ who studied these functions in the case $p=1$. The more general set of functions $T(p)$ was first introduced by Robertson $[5 ; 4]$, and in a recent paper by Robertson and Goodman [3] the sharp upper bound for $\left|b_{n}\right|$ in terms of $\left|b_{1}\right|, \cdots,\left|b_{p}\right|$ was obtained, namely for $n=p$ $+1, p+2, \cdots$,

$$
\left|b_{n}\right| \leqq \sum_{k=1}^{p} \frac{2 k(n+p) !}{(p+k) !(p-k) !(n-p-1) !\left(n^{2}-k^{2}\right)}\left|b_{k}\right| .
$$

We shall see in what follows that the size of $\left|b_{n}\right|$ is also governed by the locations of the zeros of $f(z)$ for functions of the set $T(p)$. More precisely we shall prove the following theorem.

THEOREM 1. Let

$$
f(z)=z^{q}+\sum_{n=q+1}^{\infty} b_{n} z^{n}
$$

Presented to the Society, December 29, 1950; received by the editors May 19, 1950 and, in revised form, June 15, 1950.

${ }_{1}$ Numbers in brackets refer to the references at the end of the paper. 
be a function of the set $T(p)$. Suppose that in addition to the qth order zero at $z=0$, the function $f(z)$ has exactly $s$ zeros, ${ }^{2} \beta_{1}, \beta_{2}, \cdots, \beta_{\text {, }}$, such that $0<\left|\beta_{j}\right|<1, j=1,2, \cdots$, s. Finally let the non-negative intoger $t$ be defined by

$$
q+s+t=p \geqq 1
$$

and let $m=[(t+1) / 2]$. Then

$$
\left|b_{n}\right| \leqq B_{n}, \quad n=q+1, q+2, \cdots,
$$

where $B_{n}$ is defined by

$$
\begin{aligned}
F(z) & =\frac{z^{q}}{(1-z)^{2 q+2 s}}\left(\frac{1+z}{1-z}\right)^{2 m} \prod_{j=1}^{\infty}\left(1+\frac{z}{\left|\beta_{j}\right|}\right)\left(1+z\left|\beta_{j}\right|\right) \\
& =z^{q}+\sum_{n=q+1}^{\infty} B_{n} z^{n} .
\end{aligned}
$$

When $t$ is odd or when $t=0, F(z) \in T(p)$ and the inequality (1.5) is sharp.

Theorem 1 is to some extent a generalization of a recent result [2, Theorem 2], where an expression similar to (1.6) occurs. However in [2] the coefficients could be complex, so that neither theorem includes the other.

2. The case $q=s=0$. We first prove Theorem 1 in the simple case that $f(z)$ has no zeros in $|z|<1$. For convenience we use the notation $g(z) \ll G(z)$ to mean that if

$$
g(z)=\sum_{n=0}^{\infty} a_{n} z^{n}, \quad G(z)=\sum_{n=0}^{\infty} A_{n} z^{n}
$$

then

$$
\left|a_{n}\right| \leqq A_{n}, \quad n=0,1,2, \cdots,
$$

and under these conditions we shall say that $G(z)$ is a majorant of $g(z)$.

Now suppose that $g(z)=1+b_{1} z+b_{2} z^{2}+\cdots$ belongs to the set $T(t)$ and has no zeros in $|z|<1$. We are to prove that

$$
g(z) \ll\left(\frac{1+z}{1-z}\right)^{2 m} .
$$

${ }^{2}$ Here and in the rest of the paper a $k$ th order zero appears $k$ times in the list $\beta_{1}, \beta_{2}, \cdots, \beta_{s}$, and $s$ is the number of zeros $\beta_{j}$ counted in accordance with their multiplicities. 
Since $g(z)$ is free of zeros, there is a function $h(z)=(g(z))^{1 / 2 m}$ such that

$$
h(z)=1+\sum_{n=1}^{\infty} \alpha_{n} z^{n}
$$

is regular and has no zeros in $|z|<1$, and the $\alpha_{n}$ are all real. Consequently $h(r)>0$ for $-1<r<1$. We shall see that for $|z|<1, \Re h(z)$ $\geqq 0$. Let us suppose that there is a $z_{1}$ inside the unit circle, for which $\Re h\left(z_{1}\right)<0$. If $z_{2}=\bar{z}_{1}$, then $\Re h\left(z_{2}\right)<0$ and further since $z_{1}$ is not real $z_{2} \neq z_{1}$. If we define $\phi=\arg h(z)$ so that for real $z, \phi=0$, and for $|z|<1, \phi$ is a continuous function, it is clear that with a proper choice of notation we have $\phi_{1}=\arg h\left(z_{1}\right)<-\pi / 2$ and $\phi_{2}=\arg h\left(z_{2}\right)$ $>\pi / 2$. Further, if not for $h(z)$ then for $h(-z),-\pi<\theta_{1}=\arg z_{1}<0$ and $\pi>\theta_{2}=\arg z_{2}>0$. Now consider $g(z)=(h(z))^{2 m}$ on $\Gamma$ the arc of the circle $z=\left|z_{1}\right| e^{i \theta}, \theta_{1} \leqq \theta \leqq \theta_{2}$. Since arg $g(z)=2 m$ arg $h(z)$, it is clear that arg $g(z)$ varies continuously from $2 m \phi_{1}<-m \pi$ to $2 m \phi_{2}>m \pi$ and hence $\Im g(z)$ changes sign at least $2 m+1$ times on $\Gamma$. But $g(z)$ has no zeros, so that on the full circle $z=\left|z_{1}\right| e^{i \theta},-\pi+\epsilon<\theta \leqq \pi+\epsilon$, $\Delta$ arg $g(z)=0$. Therefore on the full circle $\Im g(z)$ must change sign at least $4 m+2$ times. This is a contradiction if $g(z) \in T(t)$ where $t \leqq 2 m$. Hence if $m \geqq t / 2, \Re h(z) \geqq 0$. By Carathéodory's Theorem, $h(z) \ll(1+z) /(1-z)$ and hence (2.3) is proved. If $m$ and $t$ are integers the restriction $t \leqq 2 m$ is satisfied if $m=[(t+1) / 2]$, and this is the least integer $m$ which can be used.

3. The general case. It will be sufficient to prove (1.5) for functions $f_{p}(z)$ regular in $|z| \leqq 1$. For if $f(z)$ given by $(1.3)$ is regular in $|z|<1$ and belongs to the set $T(p)$, then for each $r, \rho<r<1$,

$$
f_{p}(z)=\frac{f(r z)}{r^{q}}=z^{q}+\sum_{n=q+1}^{\infty} b_{n}^{(p)} z^{n}
$$

is also an element of $T(p)$ and is regular in $|z| \leqq 1$. If $r$ $>\max \left\{\left|\beta_{1}\right|, \cdots,\left|\beta_{s}\right|\right\}$, the zeros of $f_{p}(z)$ will occur at $z=0$ (if $q>0$ ) and at $z=\beta_{j} / r, j=1,2, \cdots, s$. Let us denote by $B_{n}^{(p)}$ the coefficients in the power series (1.6) when $\left|\beta_{j}\right|$ is replaced by $\left|\beta_{j} / r\right|$, $j=1,2, \cdots, s$. Now $b_{n}^{(p)}$ and $B_{n}^{(p)}$ are continuous functions of $r$ and $b_{n}^{(p)} \rightarrow b_{n}, B_{n}^{(p)} \rightarrow B_{n}$ as $r \rightarrow 1$. Therefore if there were a function $f(z)$ belonging to $T(p)$ for which $\left|b_{n}\right|>B_{n}$, by taking $r$ sufficiently close to 1 , a function $f_{p}(z)$ could be constructed, regular in $|z| \leqq 1$, and satisfying the conditions of Theorem 1 , and for this function $\left|b_{n}^{(p)}\right|$ $>B_{n}^{(p)}$. 
We complete the proof of (1.5) by induction on $q$ and $s$. We have already shown that the inequality holds when $q=s=0$. Consider the three auxiliary functions,

$$
\begin{aligned}
& k_{1}(z)=\frac{1-2 z \cos \nu+z^{2}}{z}, \\
& k_{2}(z)=\frac{1-2 z \cos \nu+z^{2}}{(1+z / \beta)(1+z \beta)}, \\
& k_{3}(z)=\frac{\left(1-2 z \cos \nu+z^{2}\right)\left(1-2 z \cos \mu+z^{2}\right)}{(1+z / \beta)(1+z \beta)(1+z / \bar{\beta})(1+z \bar{\beta})},
\end{aligned}
$$

where $\nu$ and $\mu$ are real. In (3.3) $\beta$ is real and $0<\beta^{2}<1$. In (3.4) $\beta$ is not real and $0<|\beta|<1$.

On the boundary of the unit circle we have for these auxiliary functions

$$
\begin{aligned}
& k_{1}\left(e^{i \theta}\right)=2(\cos \theta-\cos \nu) \\
& k_{2}\left(e^{i \theta}\right)=\frac{2(\cos \theta-\cos \nu)}{2 \cos \theta+\beta+\beta^{-1}} \\
& k_{3}\left(e^{i \theta}\right)=(\cos \theta-\cos \nu)(\cos \theta-\cos \mu) / D_{3}(\theta, \beta)
\end{aligned}
$$

where

$$
D_{3}(\theta, \beta)=\cos ^{2} \theta+(\eta+\bar{\eta}) \cos \theta+\eta \bar{\eta}, \quad 2 \eta=\beta+\beta^{-1} .
$$

It is important to notice that in (3.6) and (3.7) the denominators are never zero for real $\theta$, and hence have constant sign. This assertion is obvious for (3.6) since $0<\beta^{2}<1$. For (3.7), we need to remark only that $D_{3}(\theta, \beta)$ as a quadratic in $\cos \theta$ has the two complex roots $\cos \theta=-\eta,-\bar{\eta}$.

Now let $f_{p}(z)$ satisfy the conditions of Theorem 1 , and suppose $q>0$, and $p>1$. Then $f_{p-1}(z)$ defined by $f_{p-1}(z)=k_{1}(z) f_{p}(z)$ is regular in $|z| \leqq 1$. Since

$$
\Im f_{p-1}\left(e^{i \theta}\right)=2(\cos \theta-\cos \nu) \Im f_{p}\left(e^{i \theta}\right),
$$

it is possible to select $\nu$ in $k_{1}(z)$ so that ${ }^{3} f_{p-1}(z) \in T(p-1)$ as indicated by the subscript. By the induction hypothesis

$$
f_{p-1}(z) \ll \frac{z^{q-1}}{(1-z)^{2 q+2 s-2}}\left(\frac{1+z}{1-z}\right)^{2 m} \prod_{j=1}^{s}\left(1+\frac{z}{\left|\beta_{j}\right|}\right)\left(1+z\left|\beta_{j}\right|\right),
$$

\footnotetext{
${ }^{3}$ Some details are omitted here. The technique is identical with that used in [3], where a more complete account may be found.
} 
and since $k_{1}^{-1}(z) \ll z /(1-z)^{2}$, the bound (1.5) follows from (3.10).

Next suppose that $s>0, p>1$ and that $f_{p}(z)$ has a real zero $\beta_{s}$, $0<\beta_{s}^{2}<1$. Set $\beta=-\beta_{s}$ in $k_{2}(z)$ and define $f_{p-1}(z)$ by $f_{p-1}(z)=k_{2}(z) f_{p}(z)$. Just as before $f_{p-1}(z)$ is regular in $|z| \leqq 1$ and for this function

$$
\Im f_{p-1}\left(e^{i \theta}\right)=\frac{2(\cos \theta-\cos \nu)}{2 \cos \theta-\beta_{s}-\beta_{s}^{-1}} \Im f_{p}\left(e^{i \theta}\right) .
$$

Again it is possible to select $\nu$ so that $f_{p-1}(z) \in T(p-1)$ as indicated by the subscript. By the induction hypothesis

$$
f_{p-1}(z) \ll \frac{z^{q}}{(1-z)^{2 q+2 s-2}}\left(\frac{1+z}{1-z}\right)^{2 m} \prod_{j=1}^{s-1}\left(1+\frac{z}{\left|\beta_{j}\right|}\right)\left(1+z\left|\beta_{j}\right|\right) .
$$

But $k_{2}^{-1}(z) \ll\left(1+z /\left|\beta_{s}\right|\right)\left(1+z\left|\beta_{s}\right|\right) /(1-z)^{2}$ and this together with (3.12) again gives the bound (1.5) for $f_{p}(z)$.

It is possible for $f_{p}(z)$ to have a complex zero which we may denote by $\beta_{s}$. Since all the coefficients are real, $\bar{\beta}_{s}$ will also be a zero of $f_{p}(z)$, and we denote this zero by $\beta_{s-1}$. Under these conditions $s>1$. Let us suppose further that $p>2$. If in $k_{3}(z)$ we set $\beta=-\beta_{8}$ and define $f_{p-2}(z)$ by $f_{p-2}(z)=k_{3}(z) f_{p}(z)$, then $f_{p^{\prime}-2}(z)$ is regular in $|z| \leqq 1$. Since

$$
\Im f_{p-2}\left(e^{i \theta}\right)=\frac{(\cos \theta-\cos \nu)(\cos \theta-\cos \mu)}{D_{3}\left(\theta,-\beta_{8}\right)} \Im f_{p}\left(e^{i \theta}\right),
$$

it is possible to select $\mu$ and $\nu$ so that $f_{p-2}(z) \in T(p-2)$. By the induction hypothesis

$$
f_{p \rightarrow 2}(z) \ll \frac{z^{q}}{(1-z)^{2 q+2 s-4}}\left(\frac{1+z}{1-z}\right)^{2 m} \prod_{j=1}^{s-2}\left(1+\frac{z}{\left|\beta_{j}\right|}\right)\left(1+z\left|\beta_{j}\right|\right)
$$

and since

$$
\frac{1}{k_{3}(z)} \ll \frac{1}{(1-z)^{4}} \prod_{j=8-1}^{s}\left(1+\frac{z}{\left|\beta_{j}\right|}\right)\left(1+z\left|\beta_{j}\right|\right)
$$

this gives again the bound (1.5).

The preceding work omits three special cases.

(a) There is a single zero at the origin, $q=p=1, s=t=0$. Set ${ }^{4}$

$$
k_{4}(z)=\left(1-z^{2}\right) / z, \quad k_{4}\left(e^{i \theta}\right)=-2 i \sin \theta .
$$

(b) There is a single real zero $\beta_{1} \neq 0, s=p=1, q=t=0$. Set

${ }^{4}$ In this case the theorem is well known and our proof is essentially that given by Rogosinski [6]. It seems worthwhile, however, to settle all three cases by a parallel argument. 


$$
k_{5}(z)=\frac{1-z^{2}}{\left(1-z / \beta_{1}\right)\left(1-z \beta_{1}\right)}, \quad k_{5}\left(e^{i \theta}\right)=\frac{-2 i \sin \theta}{2 \cos \theta-\beta_{1}-\beta_{1}^{-1}} .
$$

(c) There are two complex conjugate roots, $\beta_{1}=\bar{\beta}_{2}, s=p=2, q=t$ $=0$. Set

$$
\begin{aligned}
k_{6}(z) & =\frac{\left(1-z^{2}\right)\left(1-2 z \cos \nu+z^{2}\right)}{\left(1-z / \beta_{1}\right)\left(1-z \beta_{1}\right)\left(1-z / \beta_{2}\right)\left(1-z \beta_{2}\right)}, \\
k_{6}\left(e^{i \theta}\right) & =-i \sin \theta(\cos \theta-\cos \nu) / D_{3}\left(\theta,-\beta_{1}\right) .
\end{aligned}
$$

Now in each case $\Im f(z)$ changes sign at $\theta=0$ and $\theta=\pi$. So if $g(z)$ is defined by

$$
g(z)=k_{j}(z) f(z), \quad j=4,5,6,
$$

it is clear that $g(0)=1$, and if $\nu$ is selected properly in $k_{6}(z), \Re g\left(e^{i \theta}\right)$ $\geqq 0$. Hence in each case $g(z) \ll(1+z) /(1-z)$, and the bound (1.5) follows easily from (3.16) and the form of $k_{j}(z)$. This completes the proof of (1.5).

Corollary. Let $f(z)$ satisfy the conditions of Theorem 1, and let $F(z)$ be given by (1.6), then for $0 \leqq r<1$

$$
\left|f^{(i)}\left(r e^{i \theta}\right)\right| \leqq F^{(j)}(r), \quad j=0,1,2, \cdots .
$$

This is a trivial consequence of Theorem 1, since all the coefficients in $F(z)$ are positive.

4. Proof that the bound is sharp. We shall prove that if $t$ is zero or an odd integer, then the extremal function $F(z)$ given by (1.6) belongs to the set $T(p)$.

It was proved in [2] that if $t=0$, then $F(z)$ is $p$-valently starlike, and in this case it obviously belongs to the set $T(p)$.

Now let $t$ be odd. Then $m=[(t+1) / 2]=(t+1) / 2$. Further, for simplicity let $0<\beta_{j}<1, j=1,2, \cdots, s$, and let $2 \eta_{j}=\beta_{j}+\beta_{j}^{-1}$. The extremal function has the form

$$
F(z)=\frac{z^{q}}{(1-z)^{2 q+2 s}}\left(\frac{1+z}{1-z}\right)^{t+1} \prod_{j=1}^{s}\left(1+2 \eta_{j} z+z^{2}\right) .
$$

We first observe that $F(z)$ is a rational function of degree $2 q+2 s$ $t t+1$ and hence maps the complex $z$-plane onto a surface consisting of this number of sheets. A little computation shows that

$$
F\left(e^{i \theta}\right)=\frac{(-1)^{q+8+m} \sin ^{t+1} \theta}{2^{q}(1-\cos \theta)^{q+\delta+t+1}} \prod_{j=1}^{\delta}\left(\eta_{i}+\cos \theta\right) .
$$


Let $\epsilon=(-1)^{q+s+m}$ and consider $\Phi\left(e^{i \theta}\right)=\epsilon F\left(e^{i \theta}\right)$. If the identity $\sin \theta /(1-\cos \theta)=(1+\cos \theta) / \sin \theta$ is used in (4.2), it is clear that $\Phi\left(e^{i \theta}\right)$ is a decreasing function for $0<\theta \leqq \pi / 2$. Further it is obvious that for $\pi / 2 \leqq \theta \leqq \pi, \Phi\left(e^{i \theta}\right)$ is also decreasing. Finally, since $t$ is odd, $\Phi\left(e^{i \theta}\right)$ is an even function. Hence the boundary of the unit circle is mapped by $F(z)$ into a doubly covered slit along the real axis running from the point at infinity into the origin, the direction of the slit being governed by the sign of $\epsilon$. These facts, together with the Schwarz reflection principle, show that $F(z)$ maps $|z|<1$ onto a region consisting of $q+s+m$ sheets, one sheet being slit along the real axis as described, while the remaining sheets are fully covered. Thus $F(z)$ has valence $q+s+m$ and this is less than $p$ for $t>1$.

Let us assume for convenience that $\epsilon=1$ so that the slit is on the positive real axis. The case $\epsilon=-1$ is similar and will be omitted. We shall prove that $F(z) \in T(p)$ by decomposing each circle $|z|=r>\rho$ into four circular arcs and studying the behavior of $\Im F\left(r e^{i \theta}\right)$ on each arc.

Set $S(z)=z F^{\prime}(z) / F(z) ;$ then

$$
\begin{aligned}
& S(z)=(q+s) \frac{1+z}{1-z}+2(t+1) \frac{z}{1-z^{2}}-\sum_{j=1}^{s} \frac{1-z^{2}}{1+2 z \eta_{j}+z^{2}} \\
& S\left(e^{i \theta}\right)=i\left\{(q+s) \frac{\sin \theta}{1-\cos \theta}+\frac{t+1}{\sin \theta}+\sum_{j=1}^{s} \frac{\sin \theta}{\eta_{j}+\cos \theta}\right\} .
\end{aligned}
$$

It is obvious from (4.1) that $F(z)$ is regular on $|z|=1, z \neq \pm 1$, and from (4.4) it is clear that on these $\operatorname{arcs} F^{\prime}(z) \neq 0$, and the mapping is conformal. Let $\delta>0$ be given, and let $C_{1}$ be the arc of the circle $|z|$ $=r e^{i \theta}, \delta \leqq \theta \leqq \pi-\delta$, and let $C_{3}$ be the arc of the same circle $\pi+\delta \leqq \theta$ $\leqq 2 \pi-\delta$. Let $\Gamma_{1}$ and $\Gamma_{3}$ be the images of $C_{1}$ and $C_{3}$ respectively under $w=F(z)$. Then the conformality of $F(z)$ shows that for each $\delta>0$ there is a $\rho_{1}<1$ such that for each $r$ in $\rho_{1}<r<1, \Im(w)<0$ on $\Gamma_{1}$ and $\Im(w)>0$ on $\Gamma_{3}$. Thus, on $C_{1}$ and $C_{3}, \Im F(z)$ does not change sign.

Next let $K_{2}$ and $K_{4}$ be the circles $|z+1|<\epsilon_{2}$ and $|z-1|<\epsilon_{4}$ respectively. For $j=2,4$, let $L_{j}$ be the region common to $K_{j}$ and $|z|<1$, let $C_{j}$ be the arc of the circle $|z|=r<1$ lying in $L_{j}$, and let $\Gamma_{j}$ be the image of $C_{j}$ under $F(z)$. We shall prove that if the circle $K_{j}$ is sufficiently small, then $\Gamma_{j}$ is starlike with respect to the origin. From (4.3), $S(z)$ has an isolated pole of first order at $z= \pm 1$, and hence if $\epsilon_{2}$ and $\epsilon_{4}$ are sufficiently small, $S^{-1}(z)$ is regular and univalent in $K_{2}$ and $K_{4}$ and maps each of those regions onto convex regions. But (4.4) shows that $\Re S^{-1}(z)=0$ on $|z|=1$. Hence $\Re S^{-1}(z)$ has constant sign in $L_{j}$. Examination of $(4.3)$ for real $z$ shows that in $L_{2}, \Re S(z)<0$, 
and that in $L_{4}, \Re S(z)>0$. The negative sign for $\Re S(z)$ in $L_{2}$ means that as $z$ describes $C_{2}$ in a counterclockwise direction, $F(z)$ describes $\Gamma_{2}$ in a clockwise direction.

Returning now to $F(z)$, we observe that it has a root of order $t+1$ at $z=-1$ and hence each circle $|z+1|=r<\epsilon_{2}$ maps into a curve which goes around the origin $t+1$ times. The identity $F(z)=F\left(z^{-1}\right)$ and equation (4.2) show that the arc of this circle which lies in $L_{2}$ maps into a curve which goes around the origin $(t+1) / 2$ times. Hence if $\rho_{2}$ is sufficiently close to 1 , then for each $C_{2}$ with $r>\rho_{2}, \Gamma_{2}$ is starlike with respect to the origin, and as $z$ describes $C_{2}$ in a counterclockwise direction, $\Gamma_{2}$ goes around the origin slightly less than $(t+1) / 2$ times in a clockwise direction, starting from a point in the lower half-plane and ending at the conjugate point in the upper half-plane. Therefore $\Gamma_{2}$ cuts the real axis $t$ times and $\Im F(z)$ changes sign $t$ times on $C_{2}$.

At $z=+1, F(z)$ has a pole of order $2 q+2 s+t+1$. An argument similar to the one just given shows that if $\rho_{4}$ is sufficiently close to 1 , then for $r>\rho_{4}, \Gamma_{4}$ goes around the origin, this time in a counterclockwise direction, slightly less than $q+s+(t+1) / 2$ times, and so on $C_{4}, \Im F(z)$ changes sign $2 q+2 s+t$ times.

The determination of $\delta$ and $\rho_{1}$ can now be completed. In the upper half-plane let $P_{j}(j=2,4)$ be the point of intersection of $|z|=\rho_{j}$ with the boundary of $K_{j}$ and let $Q_{j}$ be the intersection of the line segment through $P_{j}$ lying in $K_{j}$ with the circle $|z|=1$. Then $\arg P_{4}=\delta_{4}$, $\arg P_{2}=\pi-\delta_{2}$, and $\pi>\delta_{j}>0$ define $\delta_{j}$. Then $\rho_{1}$ is determined as described earlier, using $\delta=\min \left\{\delta_{2}, \delta_{4}\right\}$.

Finally let $\rho=\max \left\{\rho_{1}, \rho_{2}, \rho_{4}\right\}$. Then for $z=r e^{i \theta}, \rho<r<1, \Im F(z)$ changes sign $2 q+2 s+2 t=2 p$ times, and hence $F(z) \in T(p)$.

5. A conjecture for multivalent functions. It has been known for some time that if a function is $p$-valent in $|z|<1$, the magnitude of its power series coefficients depends on the location of its zeros [1], but as far as the author is aware no suggestion has yet been made as to just what the sharp bound is. The work of the preceding sections suggests the following conjecture.

Let $f(z)$ given by (1.3) be regular and $p$-valent in $|z|<1$, and let $f(z)$ have zeros $\beta_{j}, 0<\left|\beta_{j}\right|<1, j=1,2, \cdots, s$. Finally let the nonnegative integer $t$ be defined by (1.4) and let

$$
\begin{aligned}
F_{p}(z) & =\frac{z^{q}}{(1-z)^{2 q+2 s}}\left(\frac{1+z}{1-z}\right)^{2 t} \prod_{j=1}^{z}\left(1+\frac{z}{\left|\beta_{j}\right|}\right)\left(1+z\left|\beta_{i}\right|\right) \\
& =z^{q}+\sum_{n=q+1}^{\infty} B_{n} z^{n}
\end{aligned}
$$


then

$$
\left|b_{n}\right| \leqq B_{n}, \quad n=q+1, q+2, \cdots .
$$

It is easy to see that $F_{p}(z)$ is $p$-valent for it is a rational function of degree $2 q+2 s+2 t=2 p$ and satisfies the equation $F_{p}(z)=F_{p}\left(z^{-1}\right)$.

It may be worth noting that if $u=z /(1-z)^{2}, F_{p}(z)$ is a polynomial in $u$. Indeed it is eavy to see that

$$
F_{p}(z)=u^{q}(1+4 u)^{t} \prod_{j=1}^{s}\left(1+\left(2+\left|\beta_{j}\right|+\left|\beta_{j}\right|^{-1}\right) u\right) .
$$

The inequality (5.2) has been proved [2] in the special case that $t=0$, and $F(z)$ is starlike with respect to the origin.

\section{REFERENCES}

1. M. Biernacki, Sur les fonctions multivalentes d'ordre p, C. R. Acad. Sci. Paris vol. 203 (1936) pp. 449-451.

2. A. W. Goodman, On the Schwarz-Christoffel transformation and p-valent functions, Trans. Amer. Math. Soc. vol. 65 (1950) pp. 204-223.

3. A. W. Goodman and M. S. Robertson, $A$ class of multivalent functions, Trans. Amer. Math. Soc. vol. 70 (1951) pp. 127-136.

4. M. S. Robertson, $A$ representation of all analytic functions in terms of functions with positive real part, Ann. of Math. vol. 38 (1937) pp. 770-783.

5. - The variation of the sign $V$ for an analytic function $U+i V$, Duke Math. J. vol. 5 (1939) pp. 512-519.

6. W. Rogosinski, Über positive harmonische Entwicklungen und typisch-reele Potenzreihen, Math. Zeit. vol. 35 (1932) pp. 93-121.

UNIVERSITY OF KENTUCKY 